\title{
Structural Connectivity Analysis in Children with Segmental Callosal Agenesis
}

\author{
(D) M. Severino, (DD. Tortora, (D) B. Toselli, DS. Uccella, (D) M. Traverso, (D) G. Morana, (DV. Capra, (D)E. Veneselli, (D)M.M. Fato, and \\ (iD) A. Rossi
}

\section{ABSTRACT}

BACKGROUND AND PURPOSE: Segmental callosal agenesis is characterized by the absence of the intermediate callosal portion. We aimed to evaluate the structural connectivity of segmental callosal agenesis by using constrained spherical deconvolution tractography and connectome analysis.

MATERIALS AND METHODS: We reviewed the clinical-radiologic features of 8 patients ( 5 males; mean age, 3.9 years). Spherical deconvolution and probabilistic tractography were performed on diffusion data. Structural connectivity analysis, including summary network metrics, modularity analysis, and network consistency measures, was applied in 5 patients and 10 age-/sex-matched controls.

RESULTS: We identified 3 subtypes based on the position of the hippocampal commissure: beneath the anterior callosal remnant in 3 patients (type I), beneath the posterior callosal remnant in 3 patients (type II), and between the anterior and posterior callosal remnants in 2 patients (type III). In all patients, the agenetic segment corresponded to fibers projecting to the parietal lobe, and segmental Probst bundles were found at that level. Ectopic callosal bundles were identified in 3 patients. Topology analysis revealed reduced global connectivity in patients compared with controls. The network topology of segmental callosal agenesis was more variable across patients than that of the control connectomes. Modularity analysis revealed disruption of the structural core organization in the patients.

CONCLUSIONS: Three malformative subtypes of segmental callosal agenesis were identified. Even the absence of a small callosal segment may impact global brain connectivity and modularity organization. The presence of ectopic callosal bundles may explain the greater interindividual variation in the connectomes of patients with segmental callosal agenesis.

ABBREVIATIONS: $\mathrm{ACC}=$ agenesis of the corpus callosum; $\mathrm{HC}=$ hippocampal commissure; $\mathrm{PVC}=$ partial virtual callosotomy; seg $\mathrm{ACC}=$ segmental agenesis of the corpus callosum

S egmental agenesis of the corpus callosum (segACC) is a peculiar form of partial callosal agenesis (ACC) characterized by the absence of the central portion of the corpus callosum with disconnection between the anterior corpus callosum and the splenium. ${ }^{1,2}$ On conventional imaging, the anterior and posterior segments appear, respectively, as genual and splenial remnants, while the intermediate segment is a thin lamina, usually corre-

Received August 9, 2016; accepted after revision October 18.

From the Neuroradiology Unit (M.S., D.T., G.M., A.R.), Neuropsychiatry Unit (S.U., M.T., E.V.), and Genetic Unit of the Department of Neurosurgery (V.C.), Istituto Giannina Gaslini, Genoa, Italy; and Department of Informatics, Bioengineering, Robotics and System Engineering (B.T., M.M.F.), Università degli Studi di Genova Scuola Politecnica, Genoa, Italy.

Please address correspondence to Mariasavina Severino, MD, Neuroradiology Unit, Istituto Giannina Gaslini, via Gaslini 5, Genoa 16147, Italy; e-mail: mariasavinaseverino@gaslini.org

E Indicates article with supplemental on-line tables.

Indicates article with supplemental on-line photos.

http://dx.doi.org/10.3174/ajnr.A5043 sponding to the hippocampal commissure. ${ }^{2}$ These features were traditionally considered the result of an early destructive insult during central nervous system development. Indeed, for many years, the prevailing theory held that the corpus callosum developed in an anterior-to-posterior direction, starting with the genu, followed by the body, splenium, and finally the rostrum. ${ }^{3}$ According to this model, segACC can only be explained by an acquired disruptive event occurring after corpus callosum development has been completed. On the other hand, segACC has been described in siblings and in patients with no evidence of cerebral lesions, suggesting a malformative rather than acquired origin. ${ }^{1}$ Moreover, new theories on the bicentric origin of the corpus callosum have further supported the malformative hypothesis. ${ }^{2}$ Nevertheless, little is still known about the structural connectivity pattern and clinical-genetic phenotypes associated with this rare callosal malformation.

In past years, there has been increasing interest in the application of advanced diffusion MR imaging techniques for in vivo 
investigation of callosal malformations. In particular, diffusion tensor imaging has offered the first clues about structural white matter organization in patients with ACC, showing the connectivity pattern of Probst bundles and revealing the presence of aberrant heterotopic callosal tracts in partial ACC. ${ }^{4,5}$ Highangular-resolution DWI and probabilistic tractography, taking into account multiple fiber orientations in the same voxel, have then improved the reconstruction of WM bundles, demonstrating the wide variability of callosal connections in partial ACC. ${ }^{6}$ More recently, a further step in DWI postprocessing associated with the application of graph analysis techniques, the so-called "structural connectome," has enabled exploring the connectivity of WM networks of the human brain. ${ }^{7,8}$ This approach models the brain as a graph represented by a collection of nodes, corresponding to cortical and subcortical regions and their WM connections (edges). Several topologic metrics and properties may be defined to characterize the global and regional organization of brain networks. ${ }^{7,8}$ The structural connectivity framework has greatly improved the characterization of WM abnormalities in patients with ACC, revealing different patterns of global and local connectivity and greater interindividual variation of brain network organization compared with healthy subjects. ${ }^{9-11}$

In this study, we aimed to characterize the structural features of segACC by using constrained spherical deconvolution and probabilistic tractography and to describe the associated clinical and genetic phenotypes in 8 children with this rare malformation. Moreover, to determine the impact of segACC on large-scale brain networks, we compared network topologic properties related to integration and segregation among children with this malformation relative to healthy controls, and we applied network-based statistics to quantify connectivity differences. In particular, we hypothesized that the absence of the central callosal portion reduces the long-range global connectivity and increases the short-range local connectivity in patients with segACC. Second, we expected to find increased variability of node degree spatial distribution and correlation strengths in patients with segACC compared with controls. Finally, we postulated that the modular organization of the segACC brain is different compared with healthy controls.

\section{MATERIALS AND METHODS Subjects}

This was a single-center retrospective case-control study, performed with Gaslini Institute review board approval and parental written informed consent. We reviewed the MR imaging studies, clinical information, and genetic data of 8 children consecutively diagnosed with segACC between 2010 and 2015 (5 males; mean age at MR imaging, 3.9 years; range, 22 days to 5.11 years). Connectome analysis was performed in the 5 patients with segACC older than 2 years of age, because no major reorganization of structural modules is usually observed after this age. ${ }^{12}$ We selected 10 age-/sex-matched controls who underwent brain MR imaging with DTI for transient febrile convulsion, minor trauma, or headaches; all had normal brain anatomy, psychomotor development, and neurologic examination.

\section{Imaging Acquisition}

MR imaging was performed on a $1.5 \mathrm{~T}$ scanner with an 8-channel head coil and included 3-mm-thick axial T2WI, FLAIR, and DWI; 3-mm-thick coronal T2WI; sagittal 0.6-mm-thick 3D driven equilibrium; and 3D turbo field echo T1WI. DWI data were collected along 34 noncollinear directions by using an axial singleshot spin-echo echo-planar sequence, with b-values of 0 and $1000 \mathrm{~s} / \mathrm{mm}^{2}, \mathrm{TR}=9203 \mathrm{~ms}, \mathrm{TE}=71 \mathrm{~ms}$, section thickness $=2$ $\mathrm{mm}, \mathrm{FOV}=240 \times 240 \mathrm{~mm}$, matrix size $=128 \times 128$. Uncooperative patients were sedated during the examinations.

\section{Data Preprocessing}

The FMRIB Diffusion Toolbox 3.0 (http://fsl.fmrib.ox.ac.uk/fsl/ fslwiki/FDT) was used to correct DWI raw data for motion artifacts and eddy current distortion and to calculate the tensor and DTI metrics maps. Subsequently, we used the FMRIB Linear Image Registration Tool (FLIRT; http://www.fmrib.ox.ac.uk/) to perform linear registration between the reference $\mathrm{B} 0$ images and 3D-T1WI. Nonbrain tissue was removed by using the MRtrix3 package (https://github.com/MRtrix3/mrtrix3).

\section{Probabilistic Tractography}

Constrained spherical deconvolution and probabilistic tractography were performed on the preprocessed diffusion-weighted images by using the MRtrix3 package. ${ }^{13}$ For each subject, a wholebrain tractography consisting of 3 million streamlines was generated, by using the anatomically constrained tractography framework. ${ }^{14}$ The seeds used for generating streamlines were distributed uniformly in the gray/white matter interface. The tractograms were filtered by using the spherical deconvolution-informed filtering of tractograms, and the final result consisted of 1.5 million streamlines for each subject. ${ }^{15}$

Fiber segmentation was performed by using TrackVis 0.5.1 (www.trackvis.org) with a manually drawn multi-ROI approach, following the callosal fiber segmentation method described by Wahl et $\mathrm{al}^{6}$ (On-line Fig 1). Qualitative analysis was performed by 2 pediatric neuroradiologists with 20 and 8 years of experience, respectively, who evaluated the anatomic course of reconstructed tracts on superimposed 3D-T1WI and the presence of other brain anomalies.

\section{Cortical Parcellation and Connectivity Matrix Construction}

The 3D-T1WI was automatically segmented by using FreeSurfer 5.1.0 (http://surfer.nmr.mgh.harvard.edu/) in 84 regions (68 cortical and 16 subcortical), representing the network nodes used as seeds for connectome construction. ${ }^{16}$ To compare the structural connectivity studies of patients and controls, we modified the virtual callosotomy approach used by Owen et al, ${ }^{9}$ simulating the segACC in each matched control (partial virtual callosotomy [PVC]). In particular, in MRtrix3, we placed a manually drawn exclusion mask on the midsagittal plane over the exact callosal segment corresponding to the patient's agenetic callosum portion. The size and shape of the mask varied for each pair of matched controls on the basis of the specific type of segACC, effectively removing only the streamlines passing through the corresponding agenetic callosal segment. 
The connectome edges were calculated by using probabilistic tractography performed with MRtrix3. The tracking results of each of the seeds were masked by each of the other 83 regions, referred to as targets, to obtain a connection strength between each seed and target pair, with the total number of streamlines connecting 2 regions as the connection strength. The $84 \times 84$ connection matrices were symmetric about the diagonal, and the matrix diagonal was set to zero. The connection strength was then divided by the sum of voxels in the seed and target regions to account for differences in volumes between various cortical and subcortical regions.

Individual and consensus connectomes were created for the 3 groups of subjects: patients with segACC, controls with PVC, and controls without PVC. In particular, we thresholded each individual connectome to remove the weakest connections, setting to zero all connection strengths that were $<0.5 \%$ of the maximum strength. ${ }^{9}$ The individual connectomes were then binarized setting to 1 all the nonzero connections. For each group, a consensus connectome was obtained by averaging the thresholded matrices of all subjects in the group; subsequently, all connections that were present in $<75 \%$ of the subjects in the group were removed from the group's consensus matrix. The consensus matrices were then binarized by setting to 1 all the nonzero connections.

\section{Network Analysis}

For topology analysis, we calculated measures of structural segregation (clustering coefficient, transitivity, local efficiency, and modularity) and integration (mean degree, characteristic path length, mean normalized betweenness, global efficiency, and cost) for the binarized individual and consensus connectomes of subjects with segACC and PVC and controls, with the Brain Connectivity Toolbox (https://sites.google.com/site/bctnet).

In each group, hub nodes were separately identified for individual and consensus connectomes. A node in a single network (individual or consensus) was marked as a hub if its degree was 1 SD higher than the mean degree of its network. To test the hypothesis that the segACC brain has higher within-group variability compared with controls, we assessed the spatial distribution of node degree by transforming the degrees of the 84 nodes in each connectome into a vector and by using the correlation coefficient in a pair-wise fashion between all individuals in each group. ${ }^{9}$ To measure network similarity, we calculated the connection strength correlation coefficient between each individual connectome and its group consensus connectome, as well as between every pair of individual connectomes in each group. ${ }^{9}$

Modularity and mean participation coefficient (quantifying the diversity of intermodular connections) were calculated for each partitioning of the consensus and individual connectome. ${ }^{8}$ To evaluate the stability of modular assignment, we computed the mean HubertRand Index for each of the 3 groups: 1) between the modular assignment for each of the individual connectomes and the assignment for the consensus connectome, and 2) pair-wise between the modular assignments for the individual connectomes (Matlab R2015b; MathWorks, Natick, Massachusetts). ${ }^{9}$ The Hubert-Rand index is an adjustment of the Rand index (measuring the similarity of 2 partitions of a dataset) that takes into account the probability of both agreement and disagreement between partitions. ${ }^{17}$
To subdivide the networks into nonoverlapping modules, we applied the Louvain community detection algorithm with an iterative fine-tuning method by using the function provided by the Brain Connectivity Toolbox. The iterations were stopped when the modularity value did not change between steps.

Network-based statistics was performed to evaluate the differences in subnetworks between patients and PVC controls. ${ }^{18}$ The statistical model included a design matrix of 5 patients and 10 controls. We used a contrast vector $(1,-1)$ to test for an increase of subnetwork connectivity in controls compared with patients, and a contrast vector $(-1,1)$ to test for the opposite hypothesis. A $t$ test was applied to assess the statistical significance of betweengroup comparisons of the network metrics. For each metric, the data labels were randomly reassigned between the 2 groups and $t$ values were computed for each relabeling. A total of 5000 permutations were performed to estimate the null distribution, and associations with $t>3$ were analyzed by network-based statistics. A family-wise error rate-corrected significance level of $P \leq .01$ was used.

\section{Statistics}

To assess the statistical significance of between-group comparisons of the network metrics and the measures of connectome variability, we used a nonparametric permutation testing procedure. For each metric, the data labels were randomly reassigned between the 2 groups and $t$ values were computed for each relabeling, for a total of 5000 permutations. $P$ values were calculated on the basis of the distribution of $t$ values obtained from the permutations and were adjusted for multiple comparisons with a false-discovery rate correction. Statistical significance was set at $P<.05$. Data were analyzed by using SPSS Statistics for Mac 21.0 (IBM, Armonk, New York).

\section{RESULTS}

Clinical, genetic, and neuroradiologic characteristics of patients with segACC are described in On-line Table 1. On conventional imaging, in most patients $(7 / 8 ; 87.5 \%)$, the agenetic segment corresponded to the posterior callosal body and the splenium was hypoplastic. In 1 patient $(1 / 8 ; 12.5 \%)$, the anterior callosal body was additionally involved. Probst bundles were noted in all patients $(8 / 8 ; 100 \%)$ at the level of the agenetic callosal segment on coronal images. The anterior commissure was hypoplastic in 4 cases $(4 / 8 ; 50 \%)$. A colpocephalic appearance of the lateral ventricle was evident in 3 patients $(3 / 8,34.5 \%)$.

On constrained spherical deconvolution tractography, no callosal fiber tracts were detectable at the level of the thin lamina corresponding to the agenetic segment. Three malformative subtypes were identified on the basis of the relationship of the hippocampal commissure (HC) with the callosal remnants (Fig 1 and On-line Fig 2): The HC lay beneath the anterior callosal remnant in 3 patients (segACC type I), while in 3 other patients, it was attached to the posterior callosal remnant (segACC type II). Finally, in 2 patients, the intermediate segment of the commissural plate was made of green-coded longitudinal bundles corresponding to the fornices joined in the midline, forming the HC (segACC type III).

According to the projection areas, the intermediate agenetic

AJNR Am J Neuroradiol 38:639-47 Mar 2017 www.ajnr.org 641 


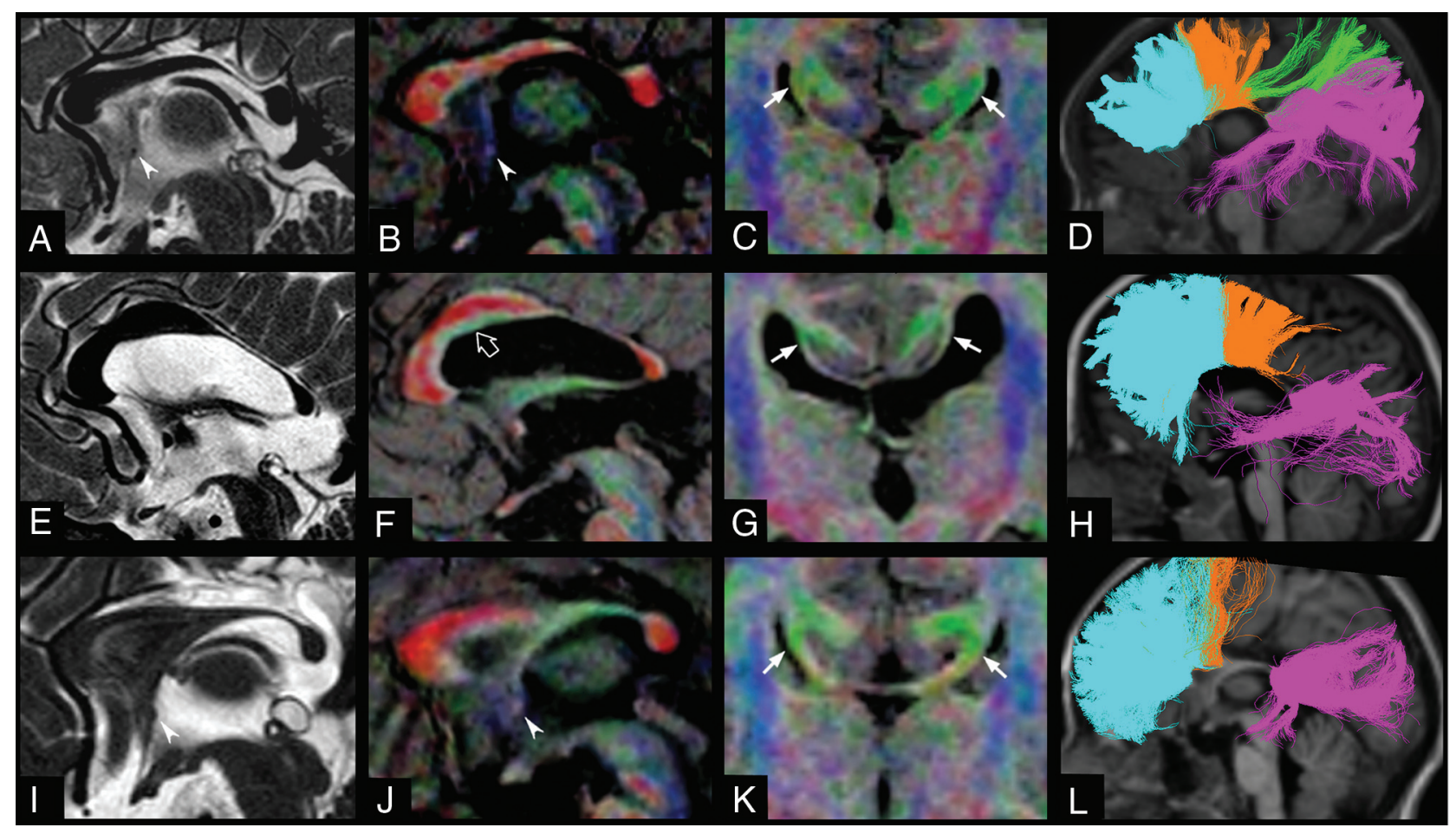

FIG 1. The 3 subtypes of segmental agenesis of the corpus callosum: SegACC type I in a 2-year-old girl (patient 3, A-D), segACC type II in a 5.4-year-old boy with Klippel-Feil syndrome (patient 5,E-H), and segACC type III in a 5.7-year-old boy with septo-optic dysplasia (patient 6, I-L). Midline sagittal T2WI driven equilibrium $(A, E$, and $I)$ and corresponding fractional anisotropy color directional maps fused with $3 \mathrm{D}-\mathrm{TWWI}(B, F$, and $/)$ reveal the focal absence of the posterior part of the callosal body in all patients. Hypoplasia of the anterior commissure may be associated, as shown in patient $3(A$ and $B$, arrowhead) and patient 6 ( $I$ and $J$, arrowhead). Note the presence of a green-coded longitudinal bundle located in the inferior part of the anterior callosal segment, corresponding to an ectopic callosal bundle in patient 5 (F,empty arrow). Coronal fractional anisotropy color directional maps fused with 3D-TTWI (C, G, and $K)$ demonstrate Probst bundles, with variable thickness, at the level of agenetic callosal segments in the 3 patients (arrows). Tractographic reconstructions of callosal fibers overlaid on $T \mathrm{WI}(D, H$, and $L)$ show the homotopic callosal connections in the 3 patients. $D$, In segACC type I, the anterior callosal remnant contains fibers connecting the anterior frontal, posterior frontal, and parietal lobes, while the posterior callosal segment contains fibers connecting the parieto-occipitotemporal lobes. In segACC types II (H) and III (L), the anterior callosal segments exclusively contain frontal callosal fibers, while the posterior callosal remnants contain fibers connecting the parieto-occipitotemporal lobes. In segACC type III ( $L)$, the intermediate segment of the commissural plate is made of green-coded bundles, corresponding to the fornices joined in the midline, forming the hippocampal commissure. The callosal fibers are colored according to their projections to specific lobar areas (ie, light blue for anterior frontal callosal fibers, orange for posterior frontal callosal fibers, green for parietal callosal fibers, and violet for occipitotemporal callosal fibers).

Table 1: Network metrics of consensus and individual connectomes

\begin{tabular}{|c|c|c|c|c|c|c|c|c|}
\hline & \multicolumn{3}{|c|}{ Consensus Connectomes } & \multicolumn{3}{|c|}{ Individual Connectomes (Mean) (SD) } & \multicolumn{2}{|c|}{$P$ Values } \\
\hline & Control & PVC & SegACC & Control & PVC & SegACC & $\begin{array}{c}\text { SegACC } \\
\text { vs Control }\end{array}$ & $\begin{array}{l}\text { SegACC } \\
\text { vs PVC }\end{array}$ \\
\hline Assortativity & 0.03 & -0.02 & 0.02 & $-0.02(0.03)$ & $-0.05(0.03)$ & $-0.02(0.04)$ & .815 & .379 \\
\hline Global efficiency & 0.45 & 0.42 & 0.38 & $0.54(0.02)$ & $0.52(0.02)$ & $0.48(0.03)$ & $.005^{\mathrm{a}}$ & $.024^{\mathrm{a}}$ \\
\hline Mean local efficiency & 0.69 & 0.70 & 0.71 & $0.77(0.02)$ & $0.77(0.02)$ & $0.75(0.04)$ & .553 & .597 \\
\hline Mean normalized betweenness & 0.02 & 0.02 & 0.03 & $0.01(0.00)$ & $0.02(0.00)$ & $0.02(0.00)$ & $.001^{\mathrm{a}}$ & $.031^{\mathrm{a}}$ \\
\hline Characteristic path length & 2.63 & 2.83 & 3.36 & $2.13(0.09)$ & $2.24(0.13)$ & $2.42(0.12)$ & $.002^{\mathrm{a}}$ & $.021^{\mathrm{a}}$ \\
\hline Transitivity & 0.38 & 0.40 & 0.40 & $0.45(0.02)$ & $0.46(0.02)$ & $0.46(0.04)$ & .555 & .904 \\
\hline Mean clustering coefficient & 0.49 & 0.51 & 0.53 & $0.56(0.03)$ & $0.57(0.03)$ & $0.57(0.04)$ & .905 & .929 \\
\hline Mean degree & 8.64 & 8.17 & 7.31 & $14.71(1.27)$ & $13.66(1.33)$ & $12.02(1.62)$ & $.005^{\mathrm{a}}$ & .054 \\
\hline Cost & 0.21 & 0.20 & 0.18 & $0.35(0.03)$ & $0.33(0.03)$ & $0.29(0.04)$ & $.005^{\mathrm{a}}$ & .054 \\
\hline
\end{tabular}

a Significant.

segment corresponded to fibers projecting to the parietal lobe in all patients with segACC and healthy controls after PVC. The hypoplastic posterior callosal remnants consistently connected the occipitotemporal lobes, while the anterior remnants always connected the frontal lobes, including the frontal associative regions and, in all except 1 case, the motor areas. In the 3 patients with segACC-type I, the anterior callosal remnants also contained splenial fibers connecting the occipitoparietal lobes. Segmental Probst bundles corresponding to the agenetic callosal segment were confirmed in all patients. These bundles merged anteriorly with the ventral callosal remnant and extended posteriorly to the parietal lobes. Callosal ectopic bundles were identified in 3 patients.

\section{Network Analysis}

Table 1 reports the topology metrics of consensus and individual connectomes. Patients with SegACC had less integrated structural connectivity compared with those with PVC and controls. No 


\begin{tabular}{|c|c|c|c|c|c|}
\hline & \multirow[b]{2}{*}{$\begin{array}{l}\text { Control (Mean) } \\
\text { (SD) }\end{array}$} & \multirow[b]{2}{*}{$\begin{array}{l}\text { PVC (Mean) } \\
\text { (SD) }\end{array}$} & \multirow[b]{2}{*}{$\begin{array}{c}\text { SegACC (Mean) } \\
\text { (SD) }\end{array}$} & \multicolumn{2}{|c|}{$P$ Values } \\
\hline & & & & $\begin{array}{c}\text { SegACC } \\
\text { vs Control }\end{array}$ & $\begin{array}{l}\text { SegACC } \\
\text { vs PVC }\end{array}$ \\
\hline Modularity & $0.38(0.02)$ & $0.41(0.03)$ & $0.44(0.03)$ & $.002^{\mathrm{a}}$ & .151 \\
\hline Mean participation coefficient & $0.39(0.05)$ & $0.31(0.07)$ & $0.28(0.04)$ & $.003^{\mathrm{a}}$ & .335 \\
\hline Mean Hubert-Rand Index (vs consensus) & $0.53(0.05)$ & $0.54(0.08)$ & $0.58(0.09)$ & .311 & .394 \\
\hline Mean Hubert-Rand Index (vs individual) & $0.63(0.03)$ & $0.61(0.06)$ & $0.68(0.02)$ & $.009^{\mathrm{a}}$ & $.017^{\mathrm{a}}$ \\
\hline
\end{tabular}

a Significant.

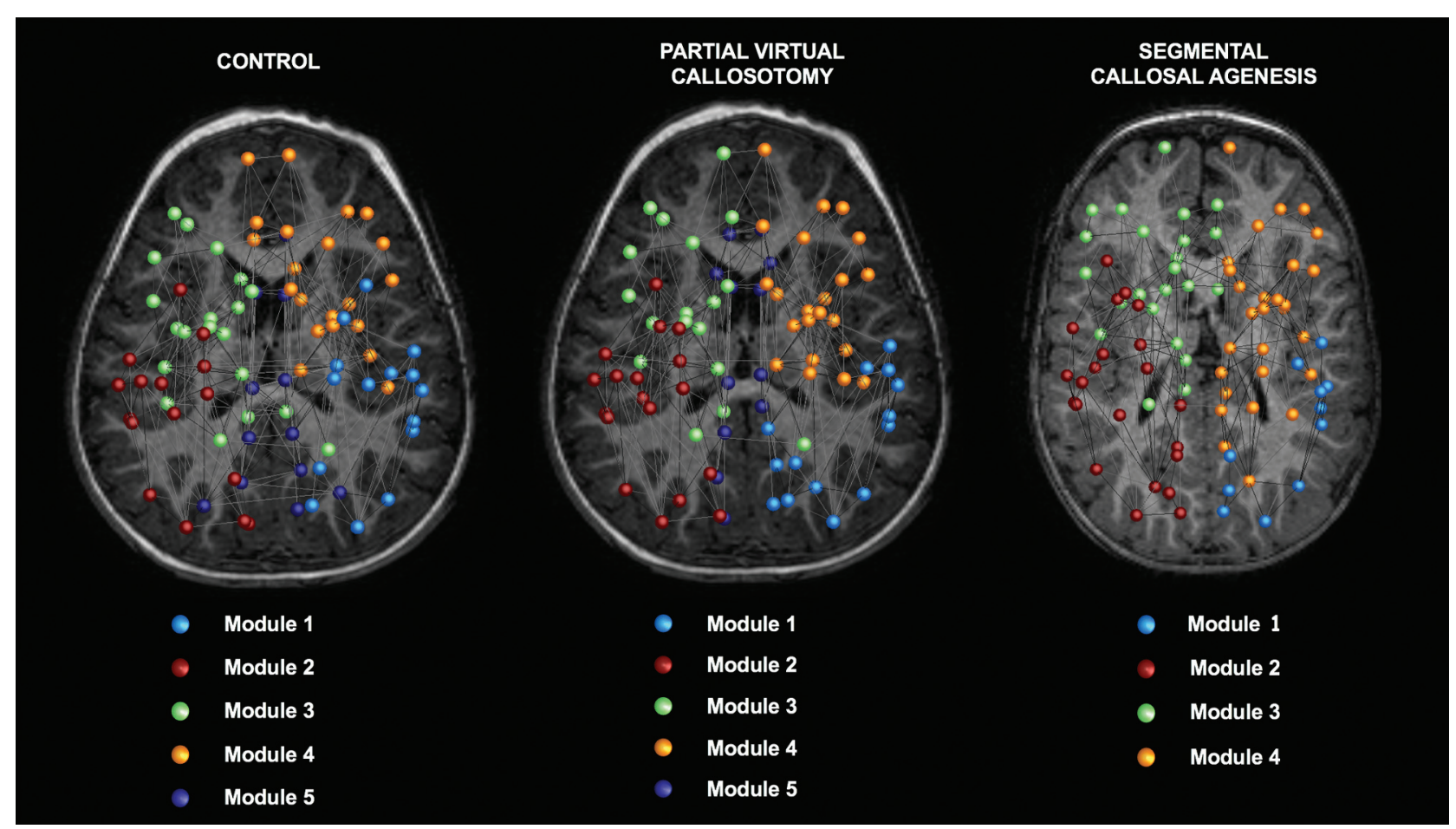

FIG 2. Module assignments for the consensus connectomes. Topology analysis of network modules reveals 5 modules for the control and partial virtual callosotomy groups and 4 modules for patients with segACC. Modules 3 and 4 largely consist of frontal nodes, while modules 1 and 2 are more posterior. Note that module 5, corresponding to the "structural core" of the network, is not present in the segACC consensus connectome. The 82 nodes are plotted with a circle colored according to the community to which it was assigned (see the legend). For the complete list of the regions included in each module, refer to On-line Tables 3-5.

significant differences in the segregation metrics were found among patients with segACC and PVC and controls.

On-line Figs 3 and 4 show the hubs found respectively for the consensus and individual connectomes. The analysis of withingroup variability revealed that the spatial distribution of node degrees was significantly more variable in segACC, as demonstrated by a lower mean correlation coefficient $(r=0.634 \pm$ $0.089)$, compared with both controls $(r=0.735 \pm 0.052)$ and subjects with PVC $(r=0.729 \pm 0.066)$ at $P<.05$.

There were no differences in the connection strength correlation coefficients between the consensus network and each individual network, indicating that the consensus connectomes represented individuals in their group to approximately the same extent. Conversely, the interindividual variability of the segACC connectome was greater than that of the control and PVC connectomes, as shown by a significantly lower consistency between individual networks $(P<.05$, On-line Table 2$)$.

The mean and SD of modularity-related metrics are provided in Table 2. Six modules were identified in the consensus network of controls and those with PVC, while only 5 were found in patients with segACC, due to disruption of the module corresponding to the structural core (Fig 2 and On-line Tables 3-5).

Network-based statistics identified 3 subnetworks of decreased connectivity in patients compared with controls (Fig 3 and Table 3).

\section{DISCUSSION}

ACC is a highly heterogeneous condition that may result from disruption of several developmental stages, from early midline telencephalic patterning to neuronal specification and axonal pathfinding. ${ }^{2,19}$ This study further supports the hypothesis that segACC is a malformation rather than an acquired condition. ${ }^{1,2}$ Indeed, in the cases here described, no signs of congenital or acquired conditions preventing commissuration, such as encephalomalacia, interhemispheric cysts, or lipomas, were noted. Moreover, although we did not identify a specific genetic phenotype associated with segACC, 2 patients had chromosomal aberrations traditionally related to callosal malformations, such as 1p36 deletion and $8 \mathrm{p}$ duplication. ${ }^{19}$ In addition, clinical-radiologic features 


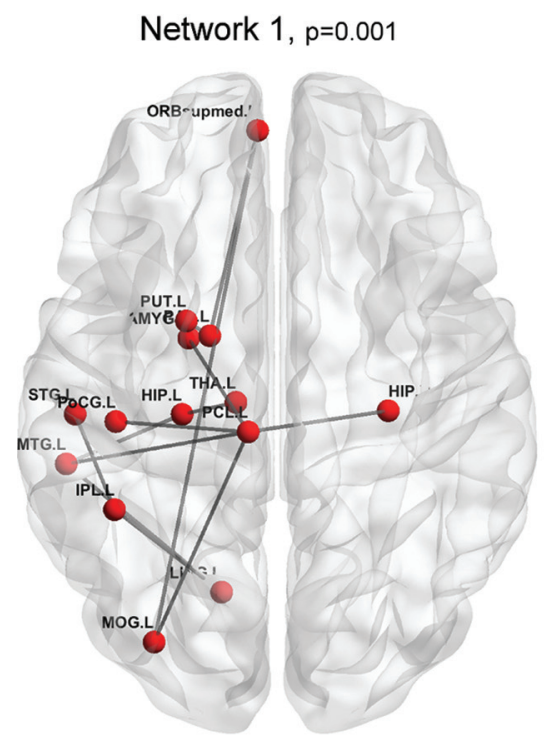

L
R

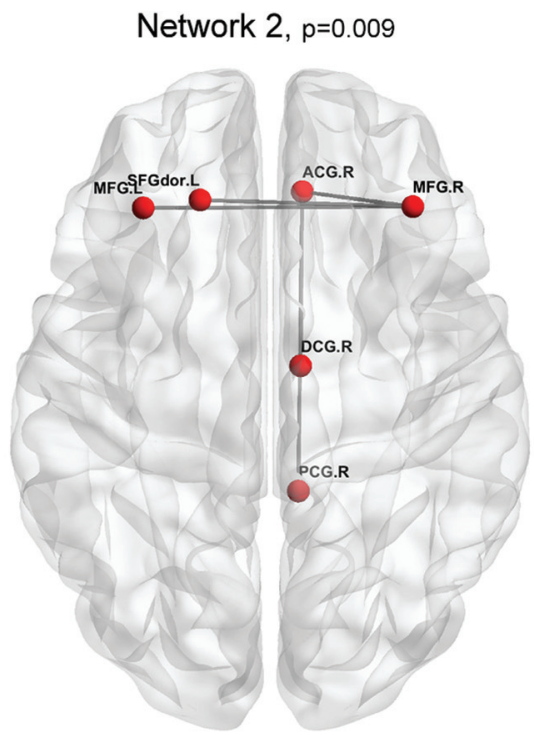

$\mathbf{L}$

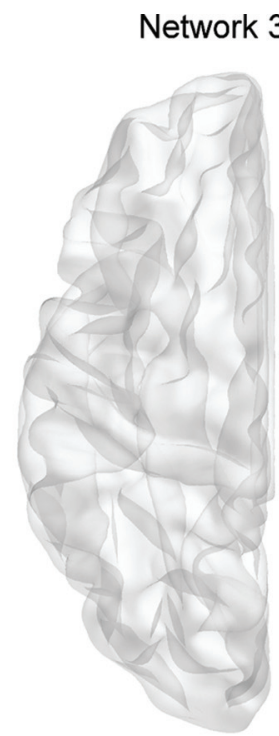

$\mathrm{R}$

FIG 3. Subnetworks with decreased connectivity in patients with SegACC compared with controls with partial virtual callosotomy. Networks 1 and 3 are prevalently intrahemispheric and involve the temporoinsular and nuclear regions, while network 2 is interhemispheric and connects the frontal lobes and right cingulum.

Table 3: Subnetworks with decreased connectivity in patients with SegACC compared with controls with partial virtual callosotomy

\begin{tabular}{|c|c|c|c|}
\hline Node 1 & Node 2 & $t$ Test Value & $P$ Value \\
\hline \multicolumn{4}{|l|}{ Network 1} \\
\hline $\mathrm{L}$ inferoparietal & L lingual & 6.24 & .001 \\
\hline$L$ lingual & L middle temporal & 5.89 & \\
\hline L paracentral & L postcentral & 5.89 & \\
\hline L hippocampus & L middle temporal & 4.16 & \\
\hline L paracentral & L amygdala & 3.61 & \\
\hline L inferoparietal & L postcentral & 3.61 & \\
\hline L paracentral & L middle temporal & 3.53 & \\
\hline L pallidum & L medio-orbitofrontal & 3.53 & \\
\hline L paracentral & R hippocampus & 3.53 & \\
\hline L hippocampus & L thalamus proper & 3.53 & \\
\hline L middle occipital & L medio-orbitofrontal & 3.53 & \\
\hline L putamen & L pallidum & 3.18 & \\
\hline L paracentral & L medio-orbitofrontal & 3.18 & \\
\hline \multicolumn{4}{|l|}{ Network 2} \\
\hline L superior frontal & $\mathrm{R}$ rostral middle frontal & 3.53 & .009 \\
\hline R posterior cingulate & $\mathrm{R}$ rostral anterior cingulate & 3.53 & \\
\hline $\mathrm{R}$ isthmus cingulate & $\mathrm{R}$ rostral anterior cingulate & 3.53 & \\
\hline L rostral middle frontal & $\mathrm{R}$ rostral middle frontal & 3.18 & \\
\hline $\mathrm{R}$ rostral anterior cingulate & $\mathrm{R}$ rostral middle frontal & 3.18 & \\
\hline \multicolumn{4}{|l|}{ Network 3} \\
\hline $\mathrm{R}$ lingual & R insula & 3.61 & .01 \\
\hline R middle temporal & $\mathrm{R}$ lingual & 3.61 & \\
\hline R middle temporal & R temporal pole & 3.53 & \\
\hline R middle occipital & $\mathrm{R}$ insula & 3.18 & \\
\hline
\end{tabular}

Note:-R indicates right; $L$, left.

of septo-optic dysplasia and Klippel-Feil syndrome were noted in 2 other patients.

Notably, the constrained spherical deconvolution tractography data on the interhemispheric connections of these patients provided further insight into the embryogenesis of segACC. Recent data on callosal formation have suggested that at about gestational weeks 12-13, the first pioneer axons from the cingulate cortex cross the commissural plate in 2 separate loci, corresponding to the anterior commissure and $\mathrm{HC}$, and then fuse to form the complete corpus callosum. ${ }^{20-25}$ This complex process is guided by 3 specialized glial structures at the corticoseptal boundary: the glial sling forming a bridge between the hemispheres and guiding the anterior callosal fibers across the midline, associated with the indusium griseum glia above and the glial wedge below..$^{23,25}$ Moreover, several guidance factors (both attractive and repulsive), cell adhesion molecules, growth factors, intracellular signaling molecules, and transcription factors, are involved in this delicate developmental phase. ${ }^{22}$ Failure in any one of these steps might explain complete agenesis or incomplete formation of the corpus callosum. Hence, at least hypotheses may be formulated regarding the origin of segACC: 1) The callosal projecting neurons corresponding to the agenetic segment did not develop; 2) all callosal projecting neurons are preserved as well as the corresponding homotopic interhemispheric connections, and the malformation results from an isolated defect of fusion of the 2 callosal segments; or 3 ) the callosal projecting neurons corresponding to the intermediate agenetic segment are preserved, but there is an additional axonal guidance defect rerouting these callosal fibers in other directions with loss of homotopic interhemispheric connections. In this study, we found that the agenetic segment most commonly corresponded to the posterior part of the callosal body, resulting in a loss of homotopic interhemispheric fibers projecting to the parietal lobes. At this level, we consistently identified segmental Probst bundles rerout- 
ing the parietal projecting fibers. Moreover, we also noted heterotopic commissural tracts in 3 patients. These findings lend support to the third hypothesis: Axonal guidance defects likely play a role in this malformation.

Most interesting, we identified 3 types of segACC, characterized by different positions of the HC and connectivity patterns of the anterior remnants. In particular, when the HC lay at the level of the intermediate agenetic segment (segACC type III) or beneath the posterior callosal remnant (segACC type II), the defect of fusion between the callosal segments lay anterior to the $\mathrm{HC}$ and the anterior callosal segments exclusively connected the frontal lobes. On the other hand, when the HC lay beneath the anterior callosal remnant (segACC type I), the defect of fusion was located posterior to the $\mathrm{HC}$ and the anterior callosal remnant also contained splenial fibers connecting the parieto-occipital lobes. These findings confirm the close embryologic relationship of the splenium with the HC, as shown by studies on mouse embryos revealing that pioneer splenial axons cross the midline, fasciculating along and between the hippocampal axons at the dorsal edge of the HC. ${ }^{23}$ However, on the basis of current embryologic theories, we could not explain why the agenetic segment was located posterior to the $\mathrm{HC}$ in patients with segACC type I. In fact, the fusion between the anterior, sling-derived callosum and the HCassociated splenium has been hypothesized to occur just anterior to the $\mathrm{HC}^{24}$; however, this theory remains to be proved ${ }^{2}$ and might indeed represent an oversimplification of a more complex mechanism in which other as-yet-unidentified factors could play a role, generating variability in the mode and location of fusion between the 2 callosal segments and thereby justifying the different patterns in our series. Future genetic studies and mouse models of segACC are needed to provide further insight regarding this unsolved question.

Finally, we used a structural connectome framework to assess WM connectivity in patients with segACC. In particular, we hypothesized that the absence of a relatively small portion of the corpus callosum, corresponding to parietal commissural fibers, could have an impact on the global and local connectivity of these patients. Indeed, recent works on structural connectivity in adults and children with ACC have shown that the absence of long-range interhemispheric callosal fibers results in a reduction of information transmission and integration between the cerebral hemispheres. ${ }^{9-11}$ Additionally, an increase of local connectivity, indicating a more segregated network organization, has been observed in these subjects, ${ }^{9,10}$ likely reflecting the profound rearrangement of cortical and subcortical connectivity within the cerebral hemispheres due to the formation of pre- and postnatal structural compensatory mechanisms, such as the Probst bundles. ${ }^{9,11}$ The topology analysis in our study partially confirmed this hypothesis even in patients with segACC, showing a less integrated structural connectivity, with reduced global efficiency, and increased path length and mean normalized betweenness.

On the other hand, no significant differences in the segregation metrics were found among segACC patients and controls, differently from the high-functioning adults with ACC studied by Owen et al, ${ }^{9}$ suggesting that local connectivity is not increased. Taken together, these findings may indicate that loss of parietal commissural fibers in segACC results in a reduction of interhemi- spheric information integration, but the compensatory WM rewiring mechanisms, represented by segmental Probst bundles and heterotopic callosal connections, are insufficient to circumvent the absence of direct interhemispheric callosal connections. In other words, while the integration decreases in segACC, a potentially compensatory increase of segregation, supporting higher order cognition and specialized processing within densely interconnected brain sub-networks, ${ }^{7,8}$ does not occur. This interpretation is supported by the results of the network-based statistics analysis in patients with segACC showing decreased connectivity in 3 subnetworks mostly related to cognitive, language, and memory functions and by the presence of psychomotor delay in all our patients. Indeed, even if deficits in higher order cognition and social skills have been described in subjects with isolated ACC, their global intelligence quotient may fall within normal limits. ${ }^{25}$ Conversely, patients with partial ACC more often present a greater degree of developmental delay or cognitive impairment, with a worse neurologic prognosis. ${ }^{25}$ Therefore, we hypothesize that a distinct anatomic connectivity substrate may contribute to the different neurocognitive profiles and neurologic prognosis of patients with partial ACC compared with neurotypical individuals with complete ACC. However, because the small sample size of this study is underpowered to detect subtler effects on the segregation metrics, larger scale investigations are needed to draw firm conclusions regarding the potential role of these connectome patterns as endophenotypes in patients with complete and partial ACC.

The second step of our analysis demonstrated that the structural connectivity of segACC subjects was also different from that of normally developing controls based on local changes in brain regions that normally serve as connectional hubs. ${ }^{12}$ In particular, both in the consensus and individual analyses, the cortical and subcortical hubs demoted or promoted from their statuses were different in the segACC group compared with the PVC group, indicating that network topology reorganization in segACC may not be explained by only the exclusion of callosal fibers from the normal brain. ${ }^{9}$ Moreover, as recently described both in adults and fetuses with ACC, ${ }^{9,11}$ we found an increased connectome variability in segACC, with increased variability in the spatial distribution of node degree and in correlation strengths in patients compared with controls. There are several anatomic substrates for this increased variability, including transient aberrant WM connections during fetal life, ${ }^{11}$ the connection variability of Probst bundles, ${ }^{5}$ the thickening of phylogenetically older commissures, ${ }^{2}$ the alternative tracts through the ventral forebrain and the dorsal midbrain midline, ${ }^{26}$ and the heterotopic interhemispheric tracts. ${ }^{5}$

Most interesting, in this study, we identified aberrant commissural tracts in 3 patients with segACC. The first aberrant tracts connecting heterotopic cortical regions in subjects with partial ACC, the "sigmoid aberrant bundles," were described by TovarMoll et al in 2007. ${ }^{5}$ These S-shaped fascicles typically connected 1 frontal lobe with the contralateral parietal lobe. Later, Wahl et al ${ }^{6}$ demonstrated the wide variability and complexity of heterotopic callosal connections in partial ACC. The functional role of these miswired fibers, however, is a matter for speculation because a direct approach to their functional performance is not available. These heterotopic callosal connections being found in patients 
with developmental delay and cognitive impairment, ${ }^{5}$ as in the present study, seems to point to a maladaptive rather than compensatory function.

The last part of our analysis focused on the modular organization of segACC consensus networks. Indeed, different modules have been described in patients with ACC compared with healthy controls. ${ }^{9,10}$ Accordingly, we found a lower number of modules and a higher Hubert-Rand Index in patients with segACC compared with those with PVC and controls. These findings suggest that neural plasticity in segACC reorganizes structural connectivity in a more stereotyped manner, with loss of a distinct posterior medial module corresponding to the network structural core. The structural core of the human brain comprises regions of the cortex that are highly connected and highly central. ${ }^{27}$ In particular, portions of posterior medial cortex, such as the posterior cingulate cortex, the precuneus, and the lateral and medial parietal cortex, are key core components known to be highly activated at rest in the default mode network. Therefore, it has been suggested that the structural core may have a central role in integrating information across functionally segregated brain regions. ${ }^{27}$ Owen et $\mathrm{al}^{9}$ demonstrated a weakened structural core in adults with ACC associated with reduced connectivity between regions of the cingulate cortex. Most interesting, the focal absence of callosal fibers connecting the parietal lobes in segACC may also lead to disruption of the structural core, therefore contributing to the impairment of large-scale brain dynamics in these patients.

The present study has several limitations, including the small sample size and the suboptimal DWI data acquired on a $1.5 \mathrm{~T}$ scanner with diffusion gradients along 34 noncollinear directions and a b-value of $1000 \mathrm{~s} / \mathrm{mm}^{2}$. Indeed, for constrained spherical deconvolution approaches, higher b-values and more diffusion gradient directions are suggested. ${ }^{28}$ On the other hand, our results are consistent with those in previous structural connectivity studies on ACC..$^{9-11}$ Moreover, the feasibility of both constrained spherical deconvolution tractography and the structural connectome based on suboptimal DWI raw data has been recently demonstrated. ${ }^{10,11,29}$ Finally, the relatively high interindividual variation of the interhemispheric connections due to the presence of ectopic callosal bundles in the patient group may have hampered the quantitative comparison of topologic metrics between graphs. However, to address this problem, we created consensus connectomes, in which all connections present in $<75 \%$ of subjects are removed from the group's consensus matrix; this step smoothed out individual variation and allowed comparison of graph metrics. Future larger scale investigations performed with better DWI acquisition schemes on $3 \mathrm{~T}$ scanners are awaited to confirm our observations.

\section{CONCLUSIONS}

We have further characterized the imaging and clinical phenotypes of segACC, describing 3 malformation subtypes and providing further insight on commissural development in these patients. Moreover, we demonstrated that even the absence of a small callosal segment may impact global brain connectivity and modularity organization of brain networks. Additionally, the presence of ectopic callosal bundles may explain the greater interindividual variation of segACC connectomes. Although direct structural connectivity does not appear to be a strict determinant of functional connectivity in healthy brains, disruption of large-scale network organization may be the anatomic substrate of neurocognitive dysfunction in children with segACC.

Disclosures: Mariasavina Severino-UNRELATED: Employment: Istituto Giannina Gaslini, Comments: neuroradiology consultant; Travel/Accommodations/Meeting Expenses Unrelated to Activities Listed: European Society of Neuroradiology, Comments: invited speaker, European Course in Neuroradiology, Diagnostic and Interventional, 14th Cycle, Module 1 (October 2016).* Andrea Rossi-UNRELATED: Consultancy: Bracco Imaging S.r.l. *Money paid to the institution.

\section{REFERENCES}

1. Raybaud C, Girard N. Anatomic MRI study of commissural agenesis and dysplasia of the telencephalon (agenesis of the corpus callosum and related anomalies): clinical correlations and morphogenetic interpretation [in French]. Neurochirurgie 1998;44(suppl 1):38-60 Medline

2. Raybaud C. The corpus callosum, the other great forebrain commissures, and the septum pellucidum: anatomy, development, and malformation. Neuroradiology 2010;52:447-77 CrossRef Medline

3. Barkovich AJ, Kjos BO. Normal postnatal development of the corpus callosum as demonstrated by MR imaging. AJNR Am J Neuroradiol 1988;9:487-91 Medline

4. Lee SK, Mori S, Kim DJ, et al. Diffusion tensor MR imaging visualizes the altered hemispheric fiber connection in callosal dysgenesis. AJNR Am J Neuroradiol 2004;25:25-28 Medline

5. Tovar-Moll F, Moll J, de Oliveira-Souza R, et al. Neuroplasticity in human callosal dysgenesis: a diffusion tensor imaging study. Cereb Cortex 2007;17:531-41 Medline

6. Wahl M, Strominger Z, Jeremy RJ, et al. Variability of homotopic and heterotopic callosal connectivity in partial agenesis of the corpus callosum: a 3T diffusion tensor imaging and Q-ball tractography study. AJNR Am J Neuroradiol 2009;30:282-89 Medline

7. Sporns O, Tononi G, Kötter R. The human connectome: a structural description of the human brain. PLoS Comput Biol 2005;1:e42 CrossRef Medline

8. Rubinov M, Sporns O. Complex network measures of brain connectivity: uses and interpretations. Neuroimage 2010;52:1059-69 CrossRef Medline

9. Owen JP, Li YO, Ziv E, et al. The structural connectome of the human brain in agenesis of the corpus callosum. Neuroimage 2013;70: 340-55 CrossRef Medline

10. Meoded A, Katipally R, Bosemani T, et al. Structural connectivity analysis reveals abnormal brain connections in agenesis of the corpus callosum in children. Eur Radiol 2015;25:1471-78 CrossRef Medline

11. Jakab A, Kasprian G, Schwartz E, et al. Disrupted developmental organization of the structural connectome in fetuses with corpus callosum agenesis. Neuroimage 2015;111:277-88 CrossRef Medline

12. Hagmann P, Sporns O, Madan N, et al. White matter maturation reshapes structural connectivity in the late developing human brain. Proc Natl Acad Sci U S A 2010;107:19067-72 CrossRef Medline

13. Tournier JD, Calamante F, Gadian DG, et al. Direct estimation of the fiber orientation density function from diffusion-weighted MRI data using spherical deconvolution. Neuroimage 2004;23:1176-85 CrossRef Medline

14. Smith RE, Tournier JD, Calamante F, et al. Anatomically-constrained tractography: improved diffusion MRI streamlines tractography through effective use of anatomical information. Neuroimage 2012;62:1924-38 CrossRef Medline

15. Smith RE, Tournier JD, Calamante F, et al. SIFT: spherical-deconvolution informed filtering of tractograms. Neuroimage 2013;67:298312 CrossRef Medline

16. Fischl B, Salat DH, van der Kouwe AJ, et al. Sequence-independent segmentation of magnetic resonance images. Neuroimage 2004;23: S69-84 Medline 
17. Hubert LJ, Baker FB. Comparisons and fitting of given classification schemes. Journal of Mathematical Psychology 1977;16:233-53 CrossRef

18. Zalesky A, Fornito A, Bullmore ET. Network-based statistic: identifying differences in brain networks. Neuroimage 2010;53:1197-207 CrossRef Medline

19. Edwards TJ, Sherr EH, Barkovich AJ, et al. Clinical, genetic and imaging findings identify new causes for corpus callosum development syndromes. Brain 2014;137:1579-613 CrossRef Medline

20. Rakic P, Yakovlev PI. Development of the corpus callosum and cavum septi in man. J Comp Neurol 1968;132:45-72 CrossRef Medline

21. Lindwall C, Fothergill T, Richards L. Commissure formation in the mammalian forebrain. Curr Opin Neurobiol 2007;17:3-14 CrossRef Medline

22. Richards LJ, Plachez C, Ren T. Mechanisms regulating the development of the corpus callosum and its agenesis in mouse and human. Clin Genet 2004;66:276-89 CrossRef Medline

23. Rash BG, Richards LJ. A role for cingulate pioneering axons in the development of the corpus callosum. J Comp Neurol 2001;434: 147-57 CrossRef Medline
24. Livy DJ, Wahlsten D. Retarded formation of the hippocampal commissure in embryos from mouse strains lacking a corpus callosum. Hippocampus 1997;7:2-14 Medline

25. Paul LK, Brown WS, Adolphs R, et al. Agenesis of the corpus callosum: genetic, developmental and functional aspects of connectivity. Nat Rev Neurosci 2007;8:287-99 CrossRef Medline

26. Tovar-Moll F, Monteiro M, Andrade J, et al. Structural and functional brain rewiring clarifies preserved interhemispheric transfer in humans born without the corpus callosum. Proc Natl Acad Sci U S A 2014;111:7843-48 CrossRef Medline

27. Hagmann $P$, Cammoun L, Gigandet X, et al. Mapping the structural core of human cerebral cortex. PLoS Biol 2008;6:e159 CrossRef Medline

28. Tournier JD, Calamante F, Connelly A. Determination of the appropriate $b$-value and number of gradient directions for high-angularresolution diffusion-weighted imaging. NMR Biomed 2013;26: 1775-86 CrossRef Medline

29. Fiori S, Poretti A, Pannek K, et al. Diffusion tractography biomarkers of pediatric cerebellar hypoplasia/atrophy: preliminary results using constrained spherical deconvolution. AJNR Am J Neuroradiol 2016;37:917-23 CrossRef Medline 